\title{
A Design of Vertical Polarized Conformal Antenna and Its Array Based on UAV Structure
}

\author{
Zhang Wei ${ }^{1}$ and Yang Junfeng ${ }^{2}$ \\ ${ }^{1}$ School of Aeronautics, Northwestern Polytechnical University, Xian 710072, China \\ ${ }^{2}$ Xian Huayu Xianxiang Aeronautical Science \& Technology Ltd., Xian 710072, China \\ Correspondence should be addressed to Zhang Wei; zhangwei_npu@163.com
}

Received 11 July 2017; Accepted 20 August 2017; Published 17 October 2017

Academic Editor: Paolo Burghignoli

Copyright (c) 2017 Zhang Wei and Yang Junfeng. This is an open access article distributed under the Creative Commons Attribution License, which permits unrestricted use, distribution, and reproduction in any medium, provided the original work is properly cited.

\begin{abstract}
The designs of conformal antenna and its array usually need to combine with different carrier structures. As for cone or cylindrical carrier, through using of invasive weed optimization (IWO), the conformal array should be the optimized design as the antenna installation space requirements of cylindrical carrier; through adopting the antenna unit miniaturization method, it designs out a conformal antenna and makes it easier to be installed and the omnidirectional circular polarized beam is also realized. As for unmanned aircraft carrier, it designs out the low profile of conformal vertical polarization antenna unit; through using the electric field integral equation (EFIE) and electric/magnetic current combined-field integral equation (JMCFIE) and multilevel fast multipole algorithm (MLFMA), it analyzes the two different characteristics of unmanned aircraft carrier on the influence of antenna performance. The results obtained through experiments and JMCFIE analysis are in good agreement; according to unmanned aircraft carrier, it also designed out the antenna in X/K-band spectrum; through IWO, the antenna array is optimized; the test results show that the array scanning angle can reach the corresponding beam direction.
\end{abstract}

\section{Introduction}

The military requirements of conformal antennas can be summarized as follows. The missile conformal antenna is designed for the guided missile based on a GPS-guided system. Airborne conformal antenna, such as Israel's "Phalcon/ Eagles," forms a total of phased-array radar and the Raytheon airborne forms a total of phased-array adaptive radar; high altitude aircraft carrier platform; conformal antennas which can realize remote warning; a wide range of goals monitoring; high resolution imaging and communications and command of rapid and stable operations. It can be seen that, for different application requirements, the antenna in the vehicle platform will be different, so in the paper, it focuses on research and analysis of conformal antennas based on cylindrical carrier and the unmanned aircraft carrier.

In the University of Illinois, the research team led by professor J. M. Jin using FEM and the domain decomposition method (DDM) makes simulation for a large-scale antenna array; at the same time, through time domain finite element method and finite difference time domain method, the complex conformal antenna on the carrier is also analyzed in detail [1-5].

Through elliptic cylinder FDTD algorithm, Mohammad et al. analyzed conformal patch antenna, and its results are coincided well with the results obtained through fullwave simulation software HFSS [6-8]; Heckler et al. adopted discrete mode matching methods to analyze the conformal microstrip antenna. Based on the adaptive integral method, the radiation characteristics of the array of microarray antennas were analyzed by Zhao and Li. He and Zhao analyzed the common form of microstrip antennas based on the elegant integral equation $[9,10]$.

Ouyang analyzed the microstrip antenna of the carrier with the combination of elegant line integral equation and the physical optics. Pathak et al. took advantage of the two different high frequency methods based on ray of carrier conformal antenna and made related analysis, and the comparison of calculation results was made [11-13].

Above research work is focused on the metal carrier, while the relevant report about the medium carrier and metalmedium compound is less. Liu et al. adopted the commercial 


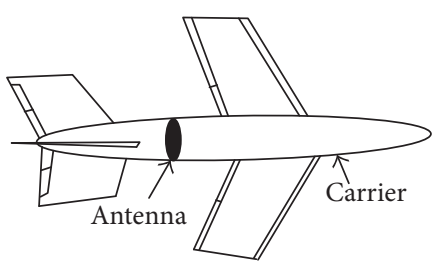

FIGURE 1: Cylindrical conformal antenna schematic diagram.

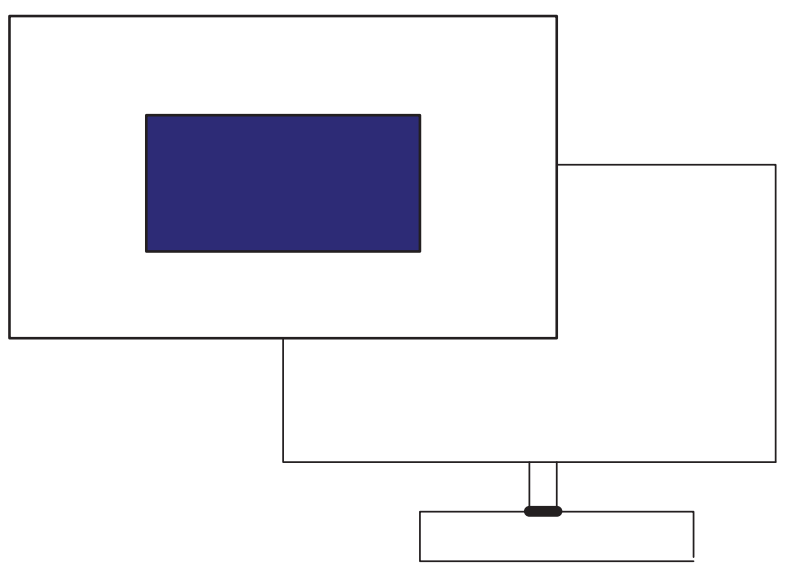

FIGURE 2: Structure of antenna feed method.

software HFSS to analyze irregular medium on the surface of the conformal antenna array [14]; the method of thin dielectric for conformal antenna is analyzed in Hou Luping's master's thesis based on the medium carrier [15].

As for conformal arrays, because the complexity of the carrier and the location of the antenna unit are different, the irregularity of the array and the direction of each antenna unit in the array are also different, so it is difficult to use the matrix theory and some conventional analytic methods to synthesize it.

\section{Conformal Antenna Design on Cylinder Carrier}

As for cylindrical beam carrier of omnidirectional circular polarized covering research, in order to realize omnidirectional coverage, antenna array is arranged on the circumference of the cylinder carrier. Obviously different curvature radius of cylindrical carrier has big influence on antenna performance, with increasing of the radius of curvature; the difficulty of realizing omnidirectional coverage will also be increased, especially in the situation of circular polarization, because it means that, as the space wave interference, the radiation pattern can form more zero depth direction points.

As shown in Figure 1, it is the schematic of conformal antenna on the cylinder; its surface circumference of the carrier is about 6.6 wavelengths of the antenna. Its length is about 9.5 wavelengths; in order to be closer to the practical application, the wing is added to cylindrical carrier; therefore the number of unknown parameters is increased; the analysis of the problem will be very complicated.

In order to reduce interference zero defect formation and realize the horizontal gain and axial ratio of omnidirectional coverage, it needs about half wavelength distribution of an antenna unit; therefore it adopts the 10 antenna array units, and each unit is fed with constant amplitude phase. Because of the needs of space installation, it requires that the whole height of antenna system should not exceed $70 \mathrm{~mm}$, so if it adopts the traditional microstrip patch, the antenna size will reach about $58 \mathrm{~mm}$, so it will bring great challenges to the design of feed method. At the same time because the antenna space is approximately 0.55 wavelengths, the mutual coupling effect between antennas is strong, so it adopts miniaturized antenna unit design; the antenna size is about $1 / 4$ wavelength. As the antenna should be conformal to the carrier, it adopts the thin and flexible medium. In order to realize the function of the array, it also designs the micro-belt power divider and the antenna integration, which will facilitate the installation of whole antenna.

2.1. Design of Antenna Unit. The conformal needs of the carrier antennas are considered; the thickness of the dielectric substrate is $1 \mathrm{~mm}$; if it adopts the single point feed form circular polarization, antenna axis is narrower than bandwidth, so the application is limited. But the double feed point method can avoid this problem, as shown in Figure 2, it is the designed two-feed antenna structure, and it adopts the Wilkinson power splitters to feed the antenna. In order to facilitate the 


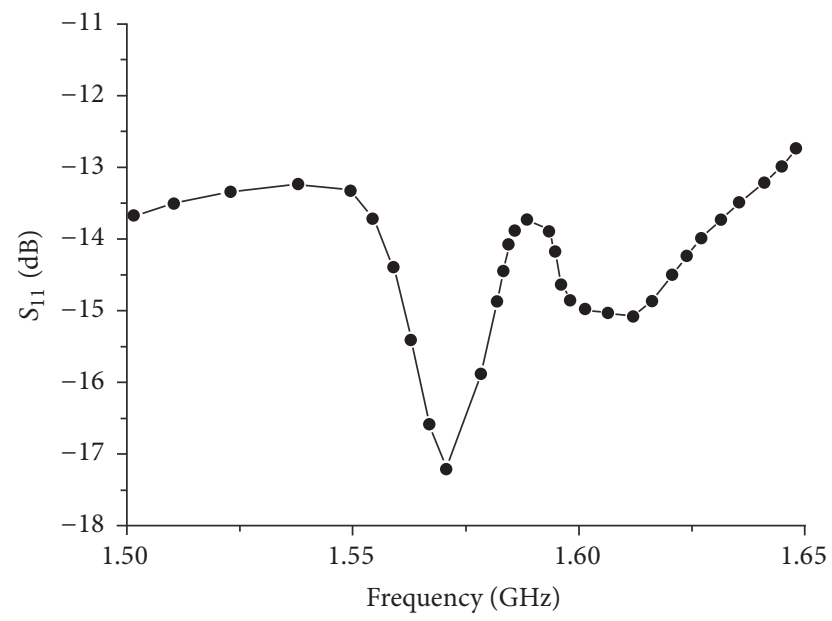

FIGURE 3: Reflection coefficient of antenna simulation unit.

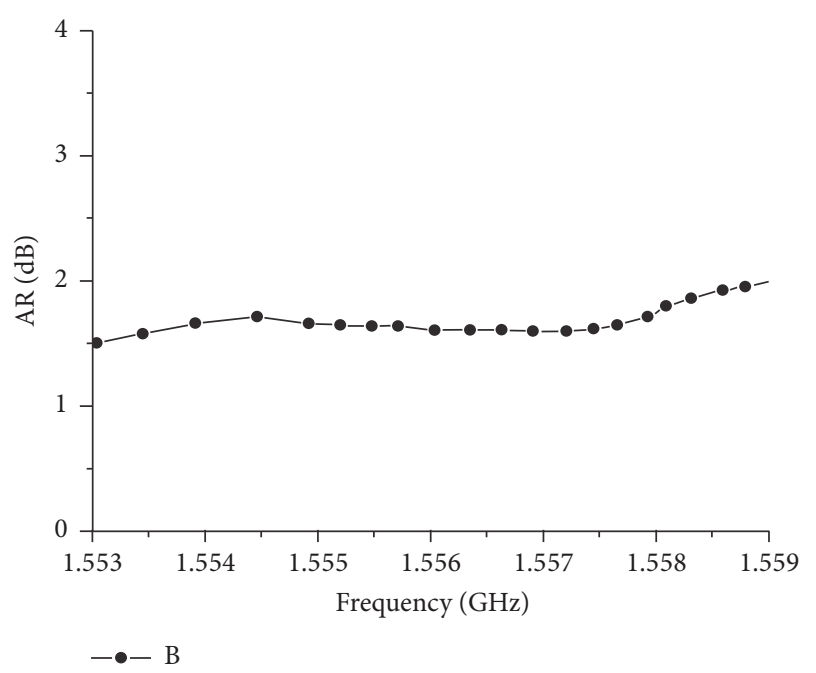

Figure 4: Axial ratio of simulation antenna unit.

power splitters to be integrated with the antenna and ensure the compact structure of entire array, it should limit the size of the power dividers; in order to avoid large width of power splitters transmission lines, the input impedance of the antenna unit is $100 \mathrm{Ohms}$. The reflection coefficients and the axis of antenna unit simulation are as shown in Figures 3 and 4 , and it can be seen that the designed antenna has a good match performance and shaft ratio performance.

2.2. Design of Antenna Array Structure. In order to minimize the size of the entire array, the antenna unit is fed by a series connection method, just as shown in Figure 5. The array can be seen as a string and a combination of structures; the designed structure is simple and easy to be processed and installed.

The simulation of designed array is performed, and the simulation of the horizontal and axial graphs is as shown in Figures 6 and 7. It can be seen that the graph of the water

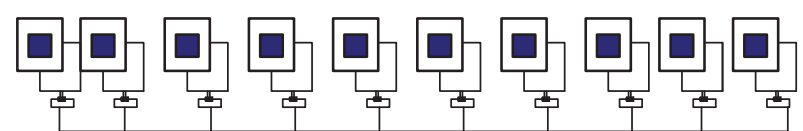

Figure 5: Series and parallel schematics of 10-element array.

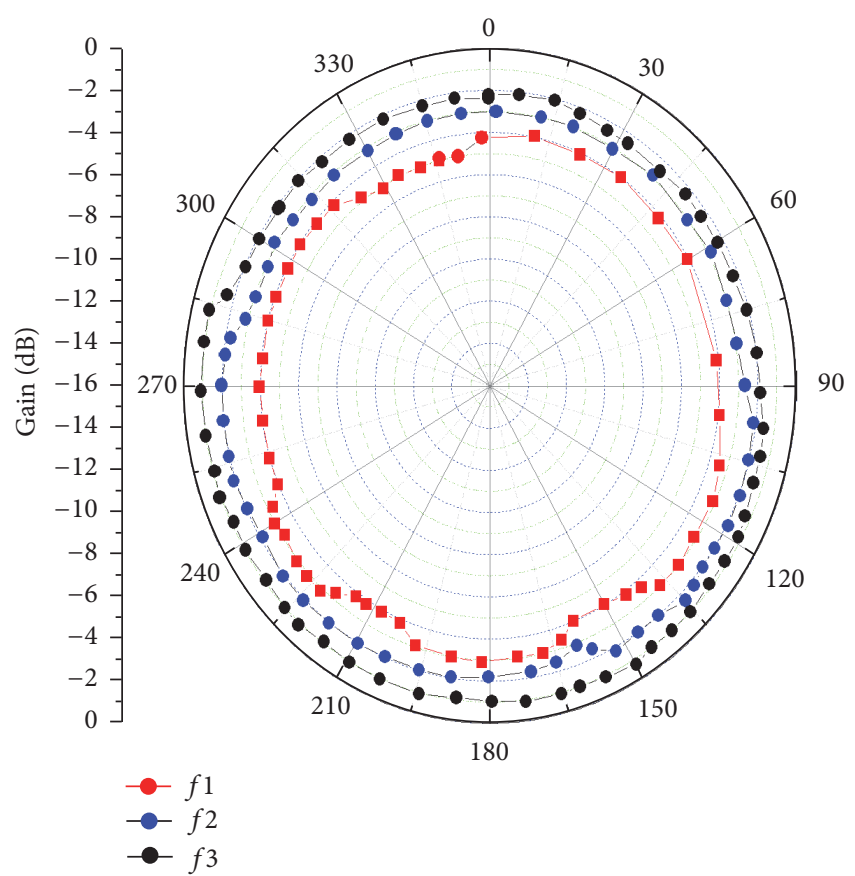

FIGURE 6: Simulation of the array radiation pattern diagram.

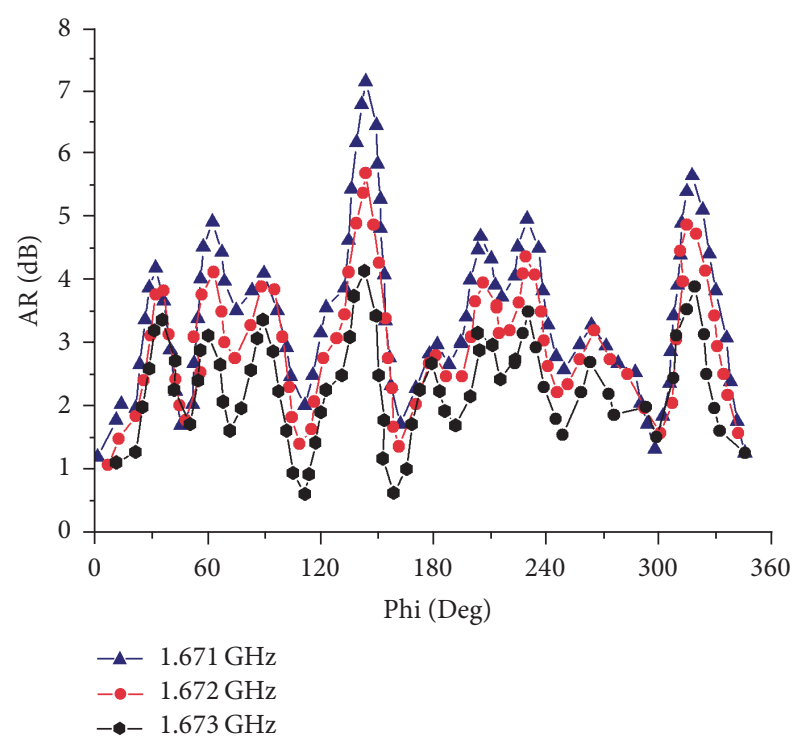

FIgURE 7: Array axis ratio in the simulation.

plane is less than $3 \mathrm{~dB}$, and the shaft ratio is less than $6.5 \mathrm{~dB}$, which can satisfy the general usage requirement.

At present, as the expanding application field of UAV, performance of UAV will affect related applications effects such as communication, detection, and investigation. The coupling of the units in the array antenna directly influences 


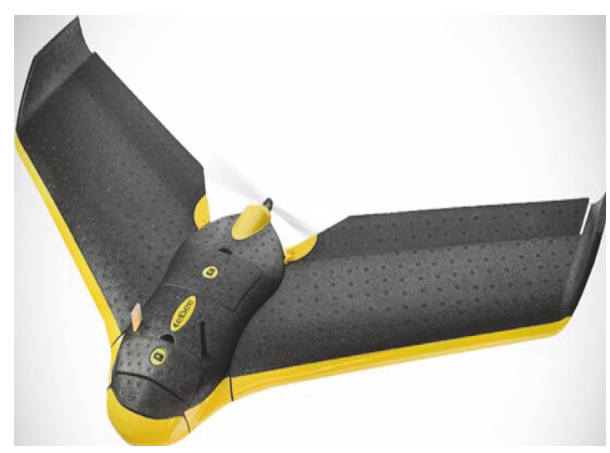

Figure 8: UAV carrier.

the performance of the array antenna system, so the decoupling is an important problem in the design of array antenna. The coupling will change the gain of the antenna and increase the sideline level and make the direction diagram distortion, so the decoupling compensation is a problem that needs to be deeply studied. Because of the common form of the airborne conformal array, the array deformation can also be a factor in the performance. In actual working environment, installed on the body surface of airborne conformal array antenna in the process of flight, due to the particularity of conformal antenna, when the body surface and airflow interaction can easily lead to conformal surface, deformation occurred and caused the amplitude and phase error and position error of the array antenna, beam deflection, the change of pattern, and so forth. And at present the conformal array antenna research is mainly concentrated on the conformal antenna unit design and radiation characteristic analysis, pattern analysis, synthesis, and optimization, as well as the conformal array antenna signal processing and so on; the study of deformation is relatively less.

Conformal array antenna used as the surface array antenna of aircraft is a kind of important application; the carrier of conformal array antenna is usually the surface of the missile, aircraft, and other mobile platforms and other complex surfaces. The geometrical shape of the rule is used to approximate the surface shape of the common carrier and then model and characterize it.

We divide the deformation of the conformal array analysis into two categories; one kind is a plane that is a nonrotating surface; the other kind of surface is the surface of the plane (such as cylindrical surface); the two kinds of the electromagnetic radiation characteristics of conformal array are different.

In the light of the carrier, more complex unmanned aircraft carrier, respectively, it designed vertical polarization antenna unit and aperture antenna array which can work in X/K-band. Figure 8 shows the drone vector model used for this section. This paper adopts electromagnetic simulation platform Ansoft HFSS to make modeling and simulation analysis of airborne antenna array, and combined with MATLAB analysis of mutual coupling, the amplitudephase error, array layout, and array deformation effect of the performance of the airborne antenna array system are studied, and the characteristics law and how to better realize

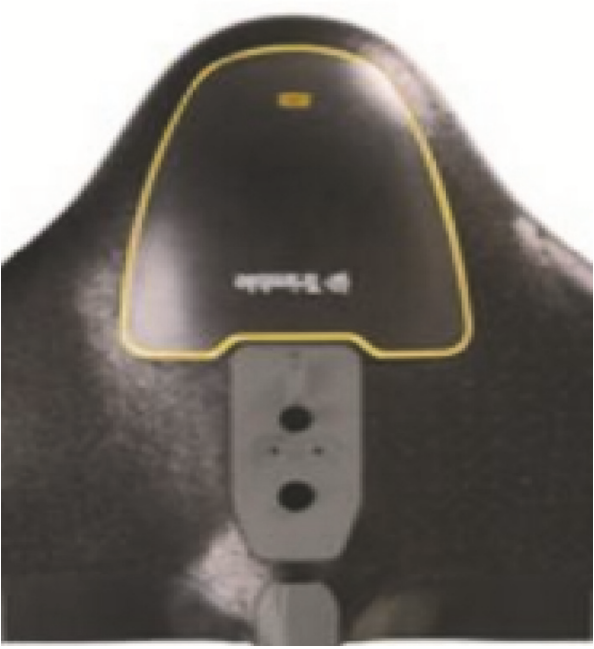

FIgURE 9: Structure of the UAV carrier antenna with carbon fiber structure.

the compensation are summarized, and the goal of the optimization of airborne antenna array system performance is achieved finally.

\section{Low Profile Vertical Polarized Antenna Design Based on UAV Structure}

As for UAV, it needs to be telemetered and send back related signal. In order to let the UAV have a wide range of flight, the designed antenna still has a high gain in low elevation. When carrier is made of metal medium, because the boundary condition of the tangential electric field is zero, if using a horizontal polarization antenna, the formation of the radiation pattern will be formed in the tangential direction of zero, and the lobe will become warped; it means that the antenna gain in low elevation is very low and thus will greatly limit the remote sensing distance of UAV.

\subsection{Aircraft Carrier Designed for Vertical Polarization Antenna} Based on Carbon Fiber Material. The UAV carrier adopts two materials commonly; one is carbon fiber, which can be approximated as a metal. The other usually adopts glass fiber reinforced plastic, which can be seen as media carriers. It carried out the research based on two different features of unmanned aircraft carrier effects on the performance of the vertical polarization antenna unit; UAV carrier with carbon fiber material is studied in the paper. Perpendicular to the plane of polarization antenna unit in the vehicle position as shown in Figure 9, it is conformal to unmanned aircraft carrier position. As for antenna radiation problem, because of the current of antenna concentrated near the antenna, there is no need to calculate the whole unmanned aircraft carrier; what needs to be calculated is whether the local structure can simulate the effect of the carrier on the antenna radiation performance. Its size is $240 \mathrm{~mm} \times 155 \mathrm{~mm}\left(5.4 \lambda_{0} \times 3.5 \lambda_{0}\right)$, through using the EFIE-JMCFIE combination of MLFMA to calculate the antenna radiation characteristics and make 


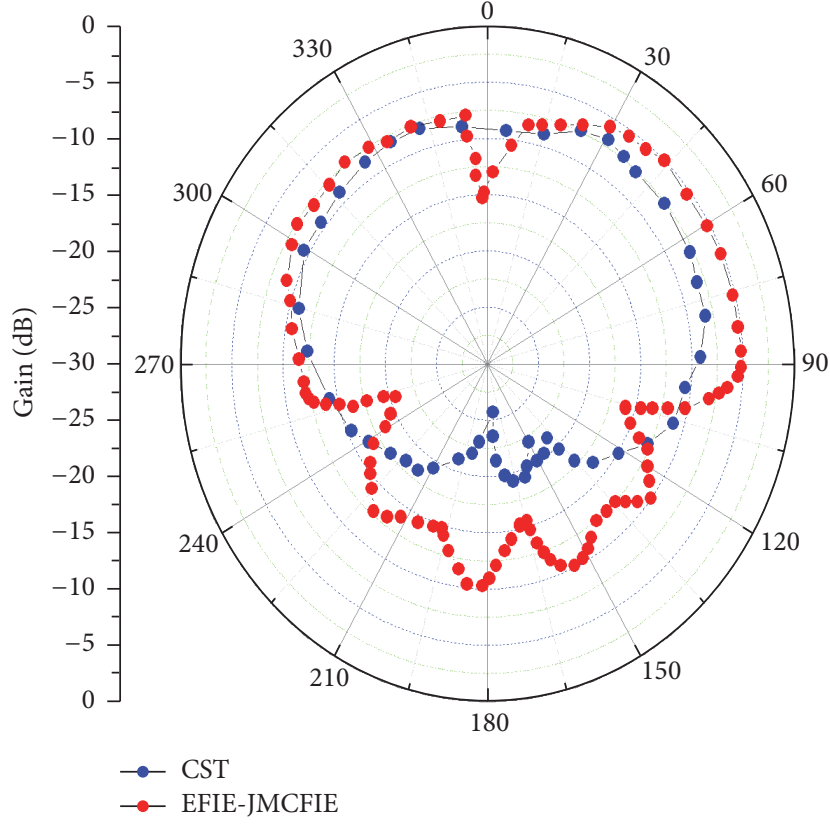

(a) $\mathrm{Phi}=0^{\circ}$

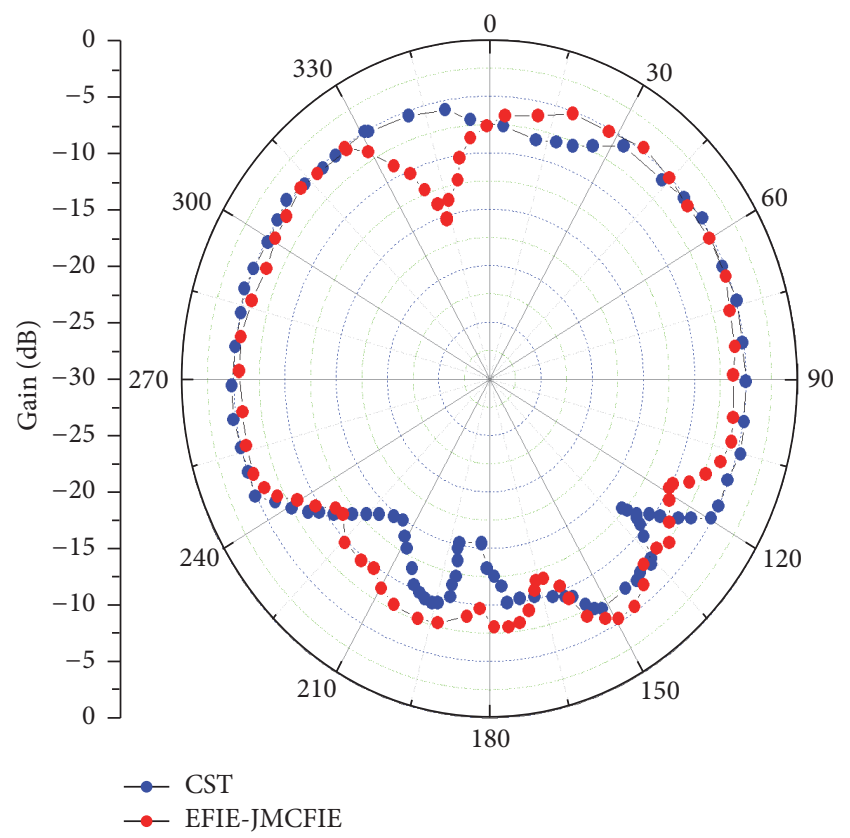

(b) $\mathrm{Phi}=90^{\circ}$

FIGURE 10: 2D direction of pitch surface.

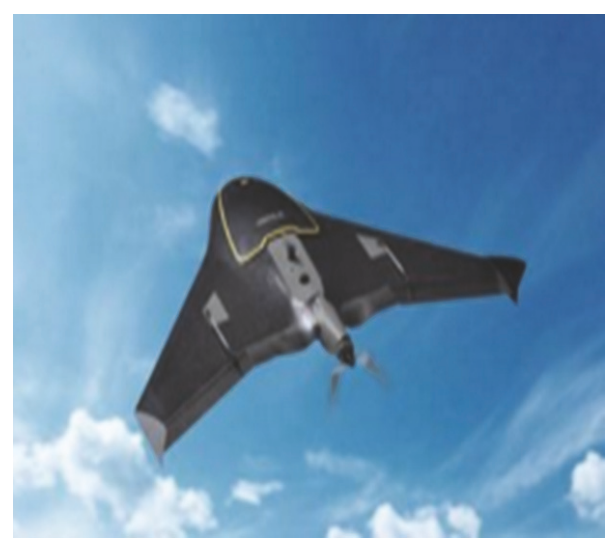

FIGURE 11: UAV and its antenna structure.

analysis of the effect of carbon fiber material carrier for antenna performance.

In Figure 10, the radiation pattern obtained through calculation is shown, and the results are compared with commercial simulation software CST; it can be seen that EFIE-JMCFIE calculation result and the calculation result of CST both have very large deviation near $0^{\circ}$. According to theoretical analysis, because it is the vertical polarization antenna, the antenna radiation pattern can produce zero trapped around $0^{\circ}$; hence calculation results of EFIE-JMCFIE are more accurate.

The antennas are processed and tested as shown in Figure 11. Testing radiation pattern is as shown in Figure 12; it can be seen that the radiation pattern obtained through testing and calculated through EFIE-JMCFIE are consistent; it also proves the validity of judgment above. However there are still some deviation between them; it is mainly because the actual material is carbon fiber material and its electrical properties are very complicated. We are unable to obtain its electrical parameters accurately, so in the calculation process metal is used in order to get approximate calculation, so there will be some deviation on its character, but it also illustrates that using metal properties instead of carbon fiber materials in simulation is feasible in practical engineering.

The influence of carbon fiber material carrier for antenna radiation performance is shown in Figure 13, as you can see that if we add antennas on the carbon fiber material carrier, antenna beam will shift to the main radiation direction; the gain will be increased in side direction; it is mainly because the antenna unit is vertical polarization antenna; thus when we place it on the carbon fiber material whose character is approximate to metal carrier, edge current will be reduced; thereby it will inhibit the upward lobe of the radiation pattern.

3.2. Design of Vertical Polarization UAV Antenna Based on Glass Reinforced Plastic Material. In this part, the influence of the antenna carrier on the antenna performance is analyzed when the carrier has medium characteristics. As shown in Figure 14, the structure of the glass reinforced plastic material is the same as the carbon fiber material carrier; the antenna is placed in the same position. As for the carrier, the dielectric constant of the glass steel body is 3 and the media loss is 0.02 , through using the radiation direction of EFIE-JMCFIE, as shown in Figure 15, which is relative to the carrier of carbon fiber. 


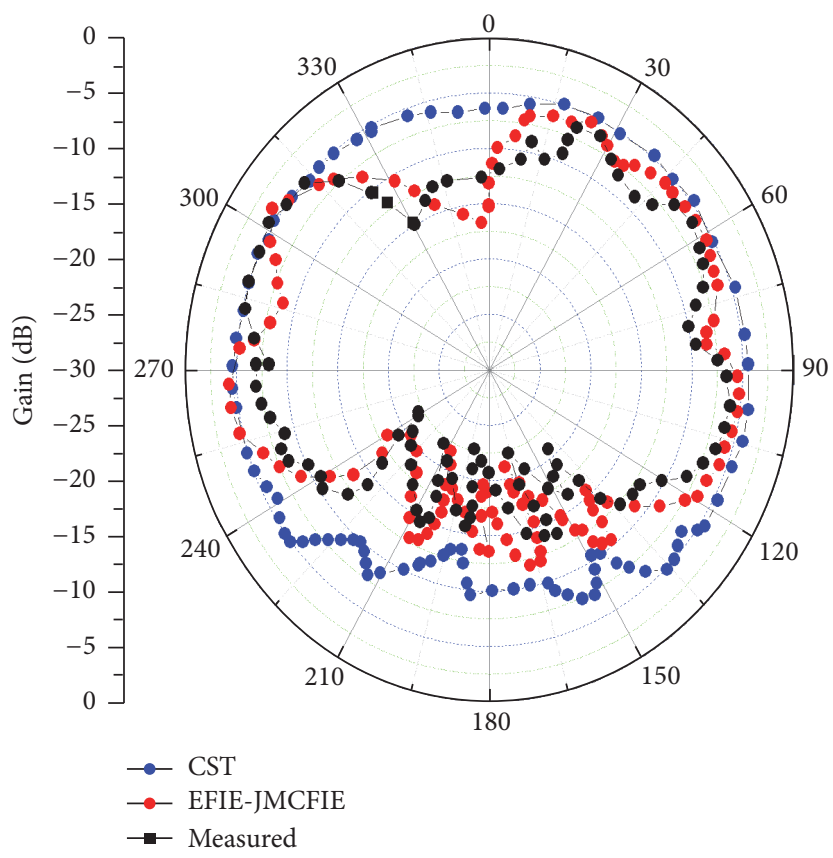

(a) $\mathrm{Phi}=0^{\circ}$

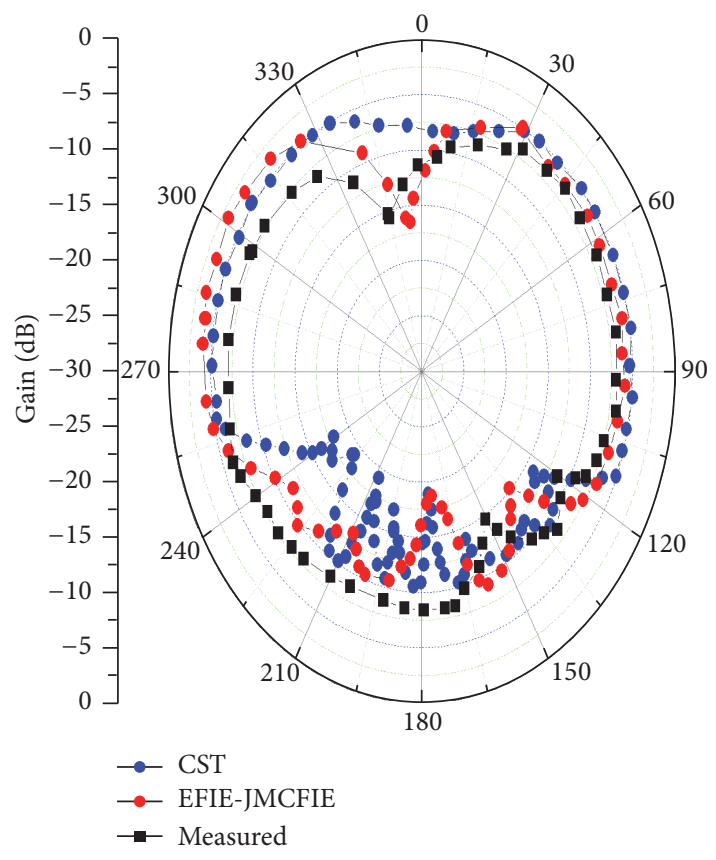

(b) $\mathrm{Phi}=90^{\circ}$

FIGURE 12: Radiation pattern of the antenna on the UAV.

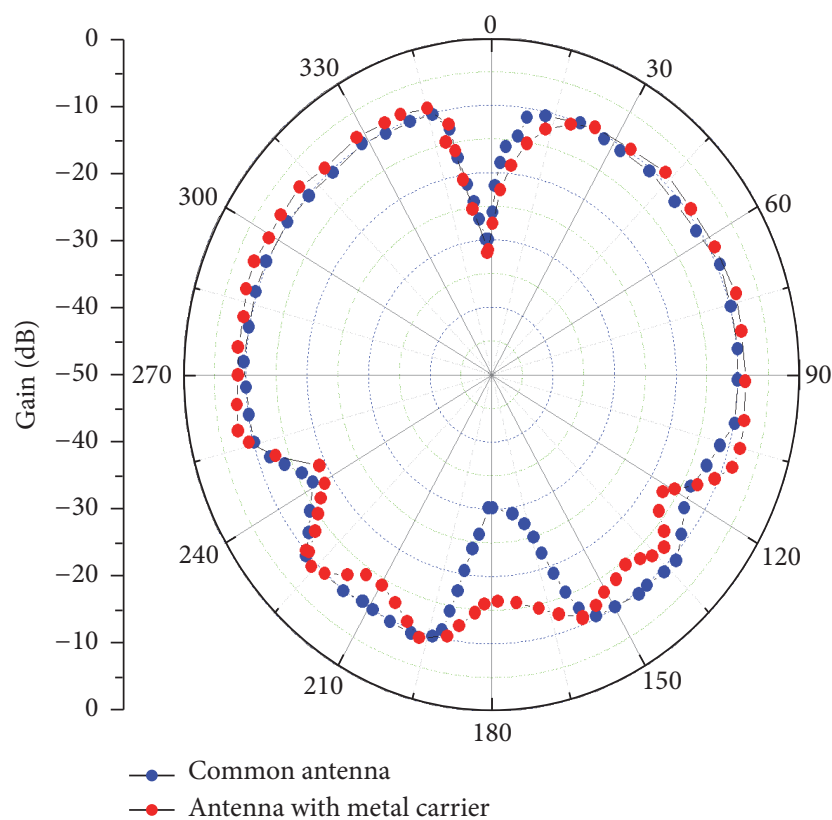

(a) $\mathrm{Phi}=0^{\circ}$

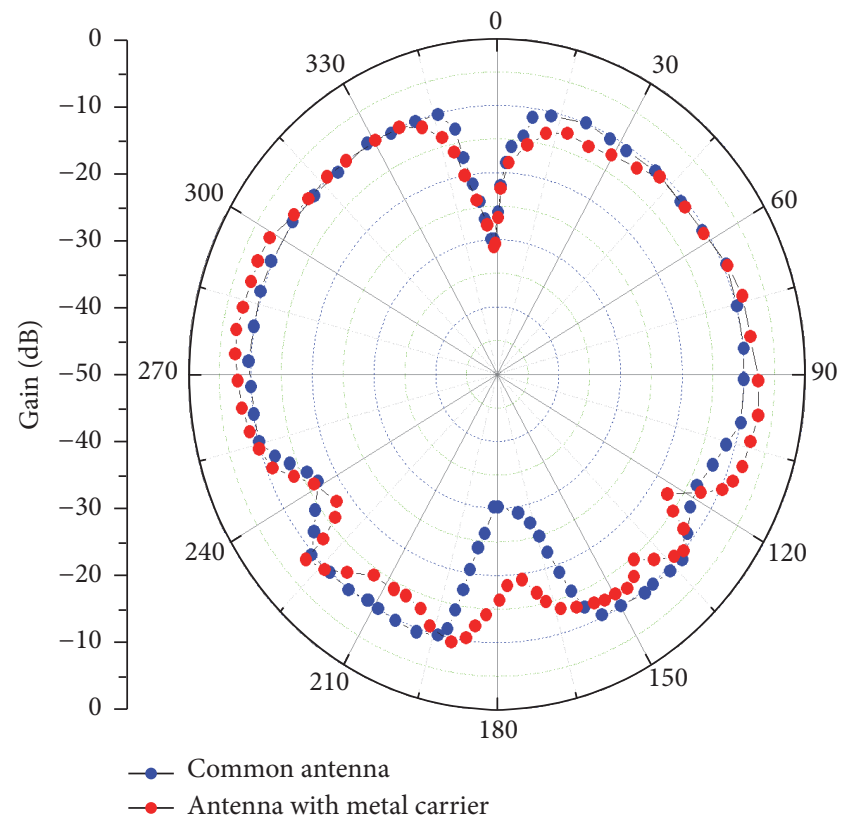

(b) $\mathrm{Phi}=90^{\circ}$

FIGURE 13: Influence of UAV material on radiation performance of vertical polarization unit.

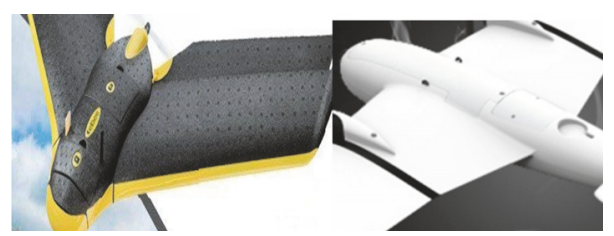

FIGURE 14: Structure of UAV antenna based on glass reinforced plastic material. 


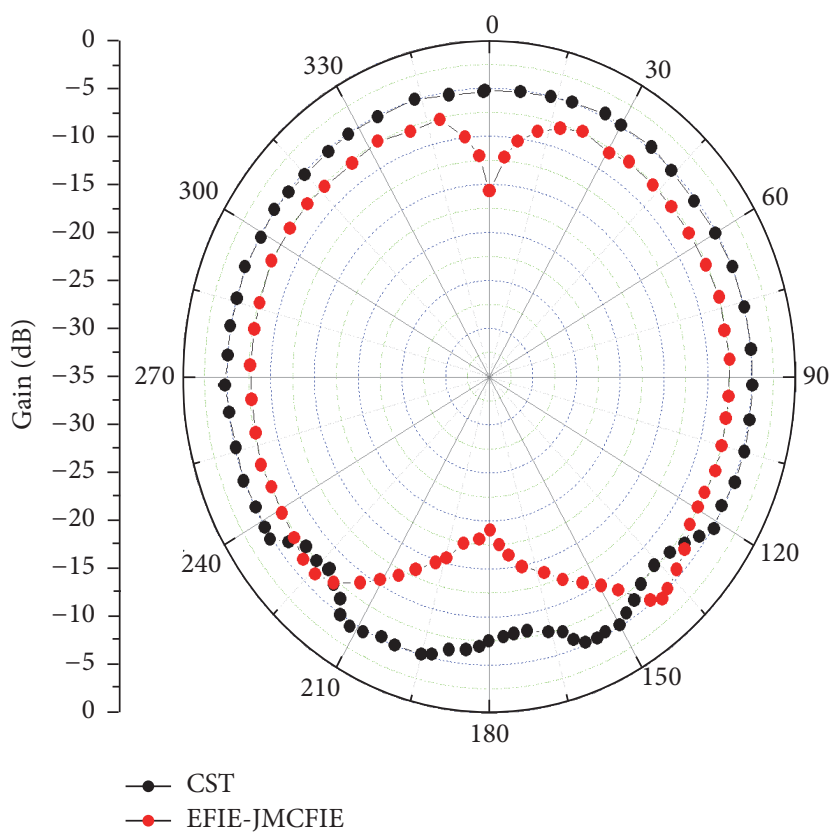

(a) $\mathrm{Phi}=0^{\circ}$

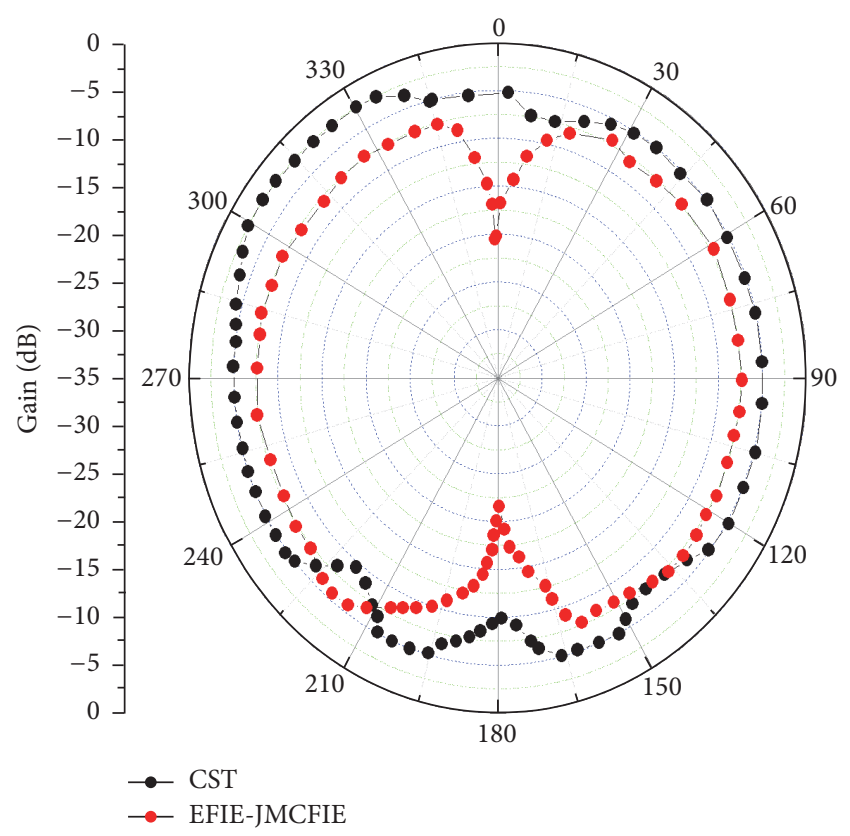

(b) $\mathrm{Phi}=90^{\circ}$

Figure 15: Two-dimensional diagram of pattern pitch surface.

The difference between calculation results and the result of CST will be bigger; it is mainly due to three reasons: one is that the carrier is medium carrier and its shape is irregular, CST subdivision of the unknown quantity is big, and subdivision needs to be applied more carefully; the other is that the CST is based on the differential equation method; it needs to set up truncated boundary conditions; different truncated boundary condition and the distance between the radiating elements will affect the radiation characteristics of analysis; the third one is that the JMCFIE method is frequency domain method, and while CST adopts time domain method, there exists Fourier transform between them.

So through time domain method and frequency domain method for calculation, the radiation pattern also can produce certain difference. This example also can illustrate that the EFIE-JMCFIE used for the analysis of the medium carrier has some advantages, because JMCFIE only need to make subdivision on the surface of the medium carrier; thus it can reduce the unknown quantity and also reduces the computational complexity. In the integral equation method, it is unnecessary to set the boundary condition.

The designed antenna is processed and tested, and the process picture is as shown in Figure 16. The radiation pattern obtained through the test is as shown in Figure 17, which shows that the results obtained by EFIE-JMCFIE are well matched with the test results. It analyzes the effect of antenna performance used on glass fiber reinforced plastic material carrier, because the antenna unit is vertical polarization antenna unit, so the glass fiber reinforced plastic material carrier had little influence upon the performance of the antenna, which is shown in Figure 18.

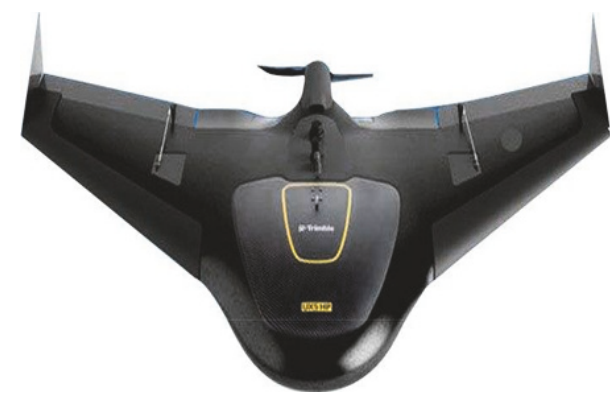

FIGURE 16: Structure of antenna processed.

3.3. Design of Antenna Array for Unmanned Aerial Vehicle. Based on the $\mathrm{X} / \mathrm{K}$ double frequency copper-caliber antenna unit, we design out the cop-caliber antenna array as shown in Figure 19. The $\mathrm{X}$-band spacing interval is $\lambda / 2$, which consisted of 8 antenna units. The array spacing of the K-band antenna is also $\lambda / 2$, which consisted of 15 antenna units. The array is also conformed to the belly of UAV carrier made of glass and steel materials. The AEP was then optimized for the array with IWO; the radiation characteristics of the array are calculated through using EFIE-JMCFIE and being combined with MLFMA. It optimizes the feed phase of the array, which can allow the arrays to scan at the required beam points.

When the optimization of the X-band antenna array is finished, the feed phase distribution of each unit is as shown in Table 1 . The optimized phase distribution method is used to calculate the array radiation pattern; the results of the calculation are as shown in Figure 20; the results of 


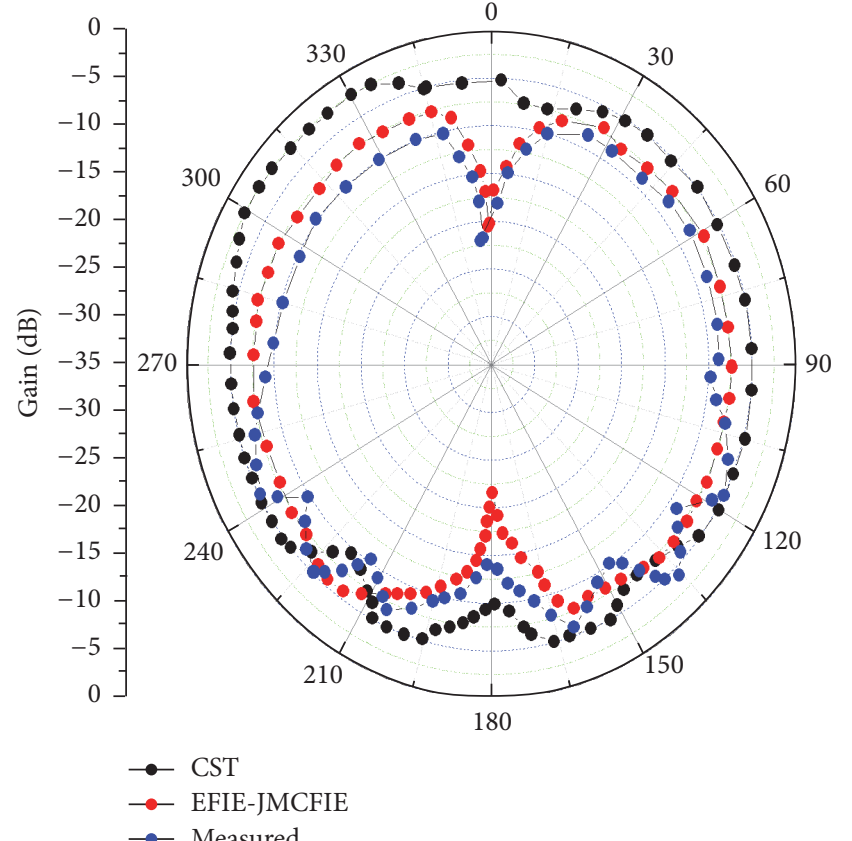

(a) $\mathrm{Phi}=0^{\circ}$

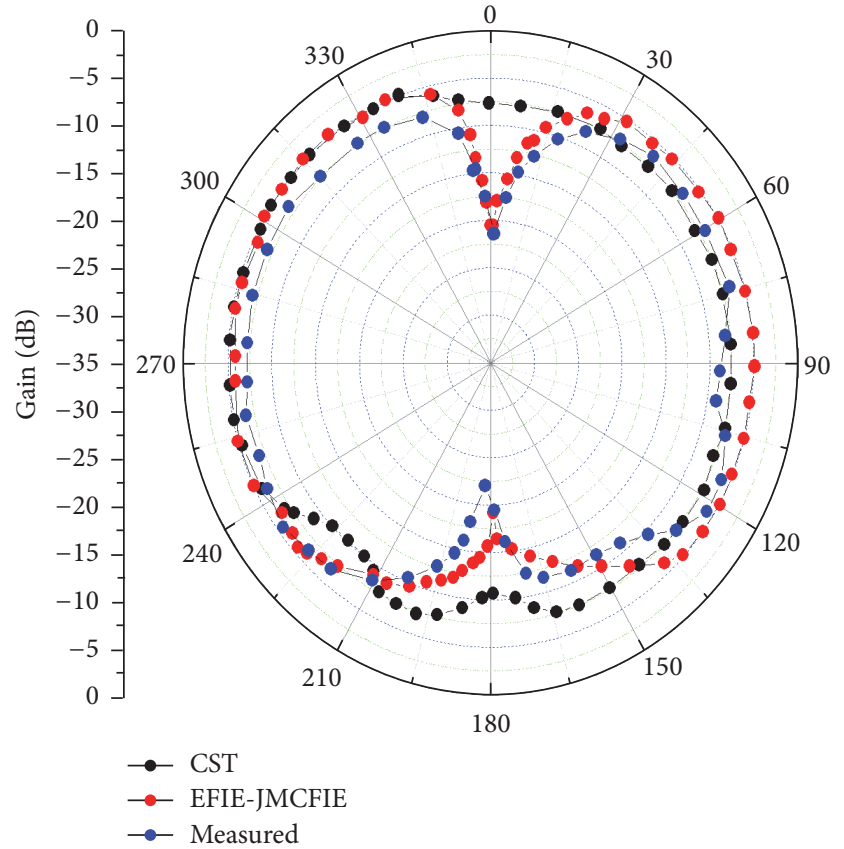

(b) $\mathrm{Phi}=90^{\circ}$

FIGURE 17: Radiation pattern of UAV.

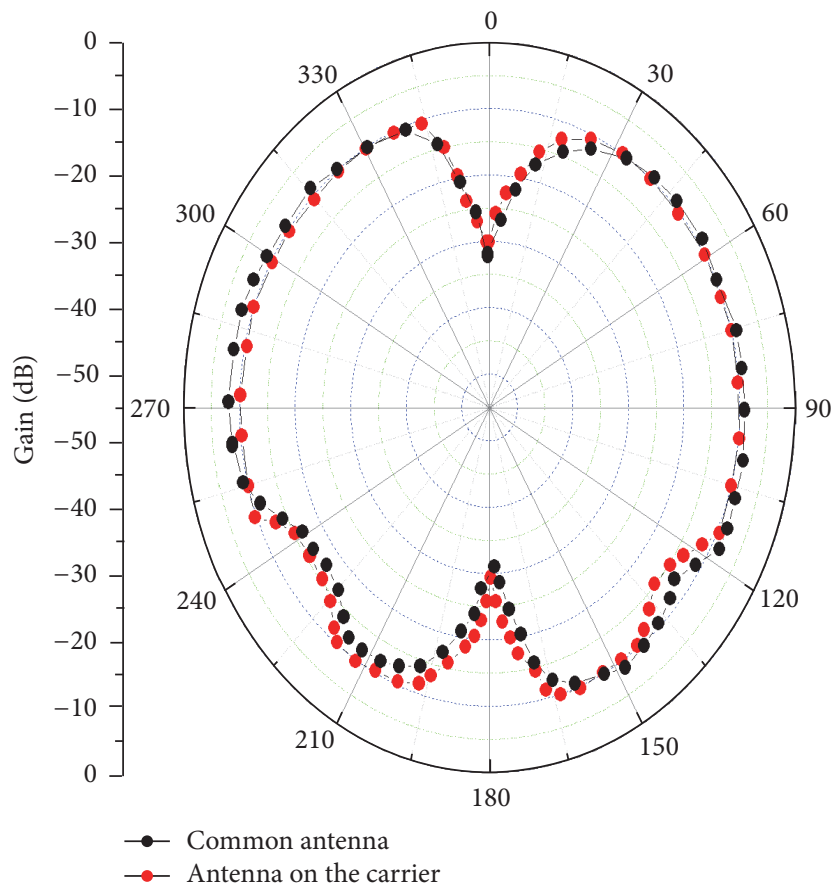

(a) $\mathrm{Phi}=0^{\circ}$

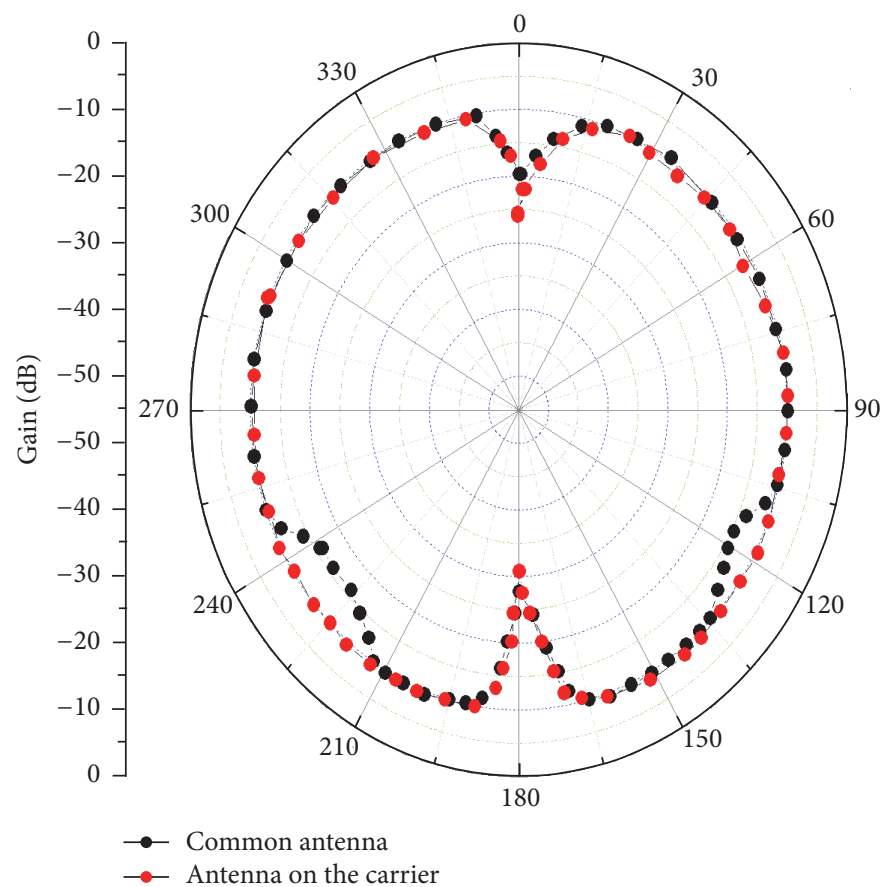

(b) $\mathrm{Phi}=90^{\circ}$

FIGURE 18: Influence of glass reinforced plastic material vertical polarization antenna.

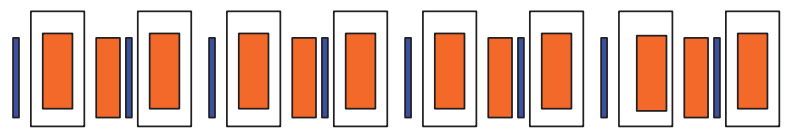

FIGURE 19: Structure of aperture antenna array. 


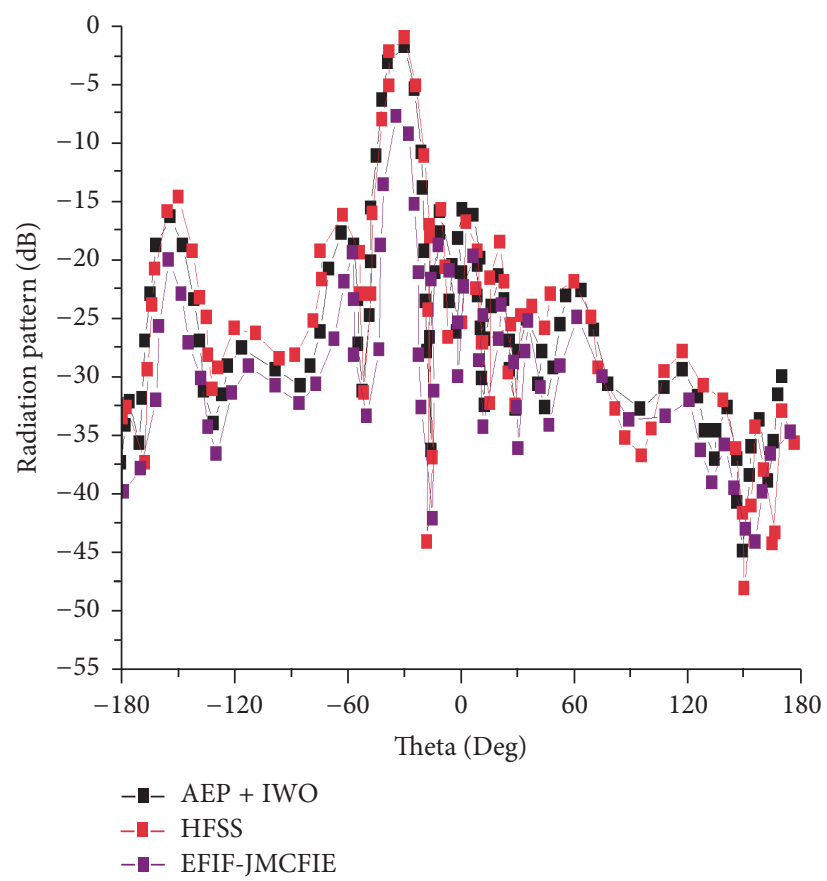

(a) Scanning angle is $-30^{\circ}$

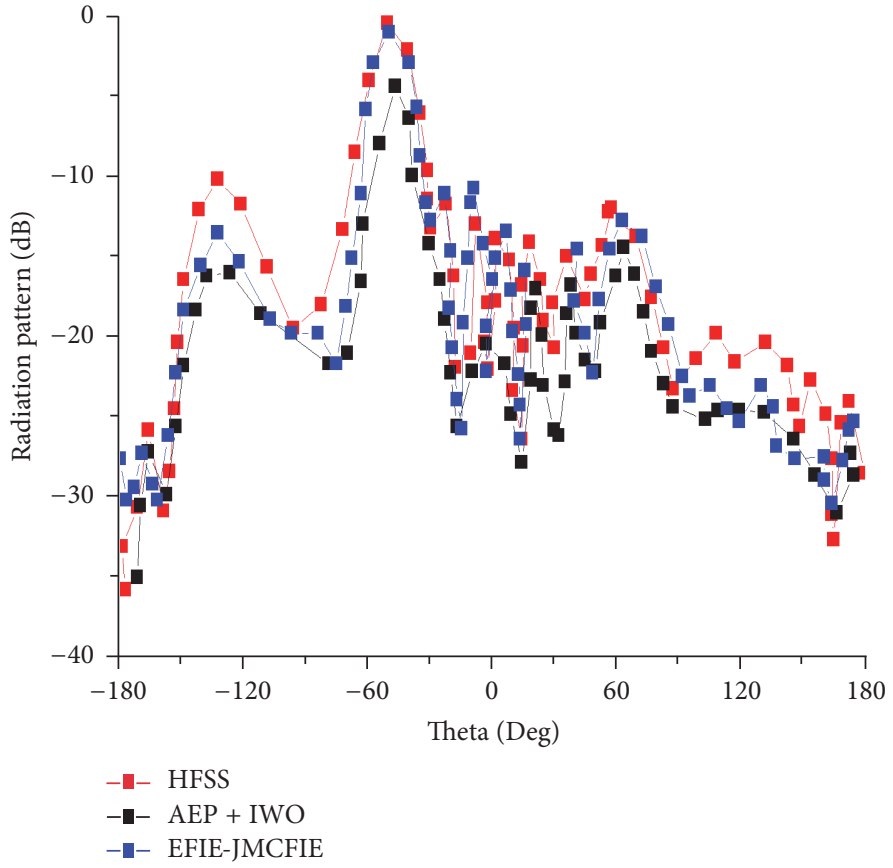

(b) Scanning angle is $30^{\circ}$

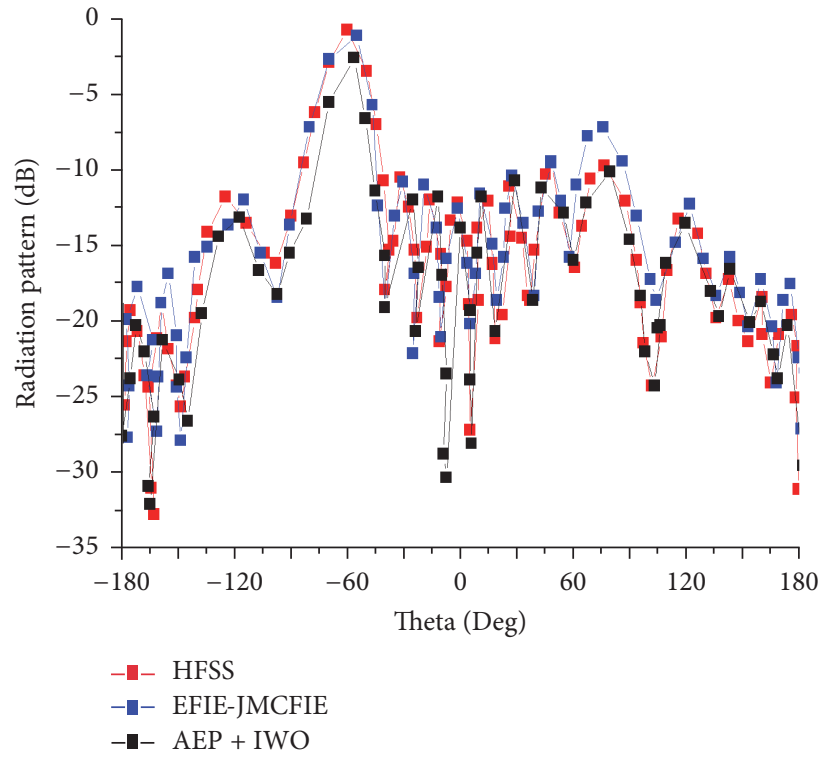

(c) Scanning angle is $60^{\circ}$

FIgURE 20: Optimized X-band array direction map.

JMCFIE calculation and active direction map superposition principle can be seen; the result of the simulation software HFSS is in anastomosis of main lobe, but there are some deviations in the side-lobe region; this is mainly due to the different algorithms of numerical error. Obviously, through using the optimization phase distribution method to feed antenna array, the array beam can scan at the specified point; JMCFIE equations in the analysis of the conformal array have more excellent performance. On the optimized K-band antenna array feed phase distribution just as shown in Table 2, it also adopts the EFIE-JMCFIE and MLFMA to make its radiation characteristics analysis; calculation result of the radiation direction of drawings is also as shown in Figure 21.

We make related testing and processing on the designed aperture array; processing of physical diagram is as shown in Figure 22; radiation direction of X-band test diagram is shown in Figure 23; test results and the simulation results agree well in the main lobe area, while there are some differences in the side-lobe region. Since the laboratory's work integrator and phase shift are unable to work in $\mathrm{K}$-band, the radiation direction of the K-band array is not tested in this part. 


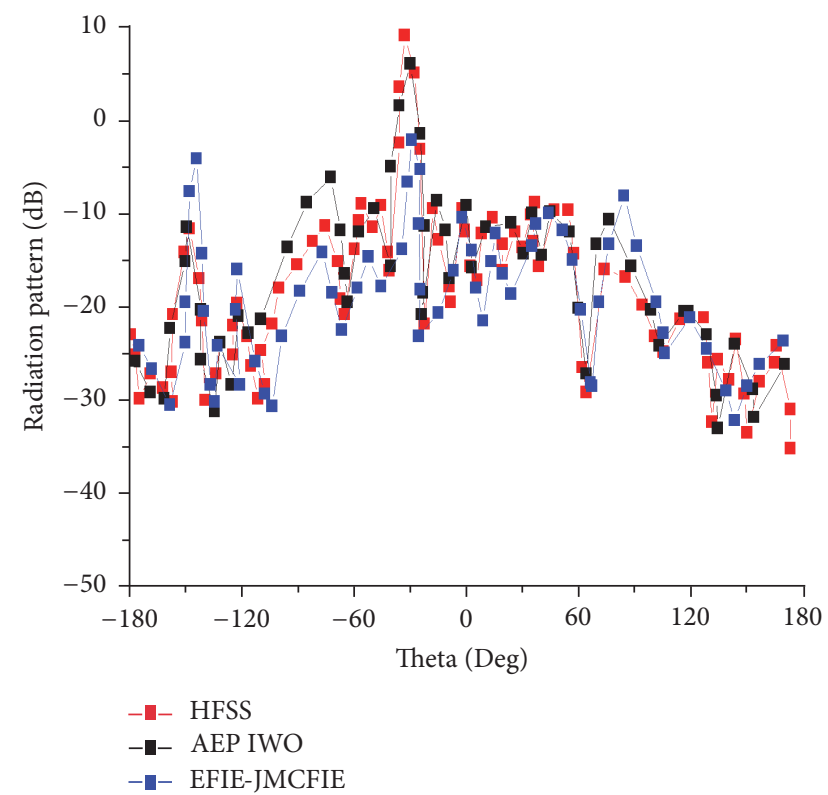

(a) Scanning angle is $-30^{\circ}$

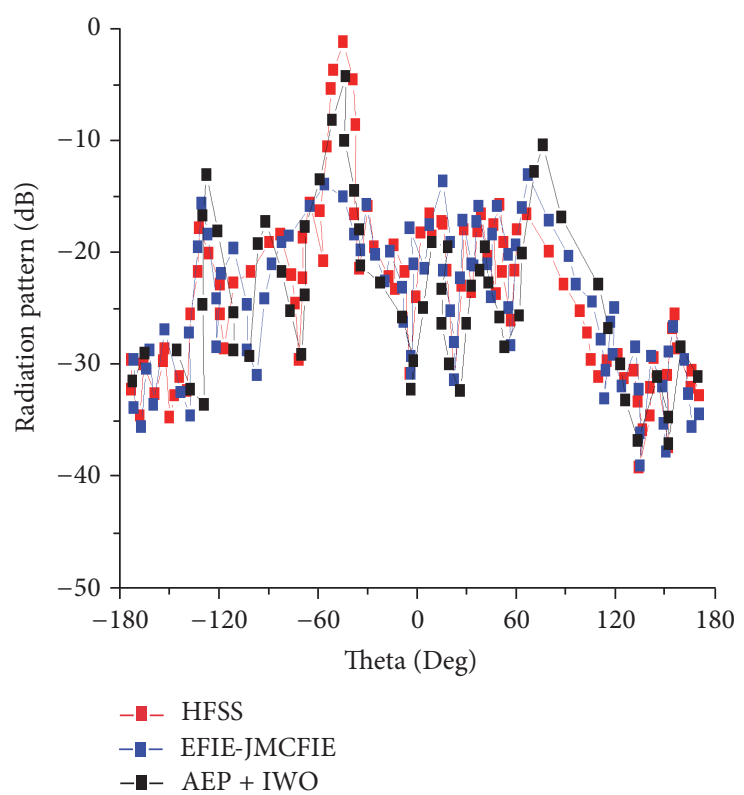

(b) Scanning angle is $30^{\circ}$

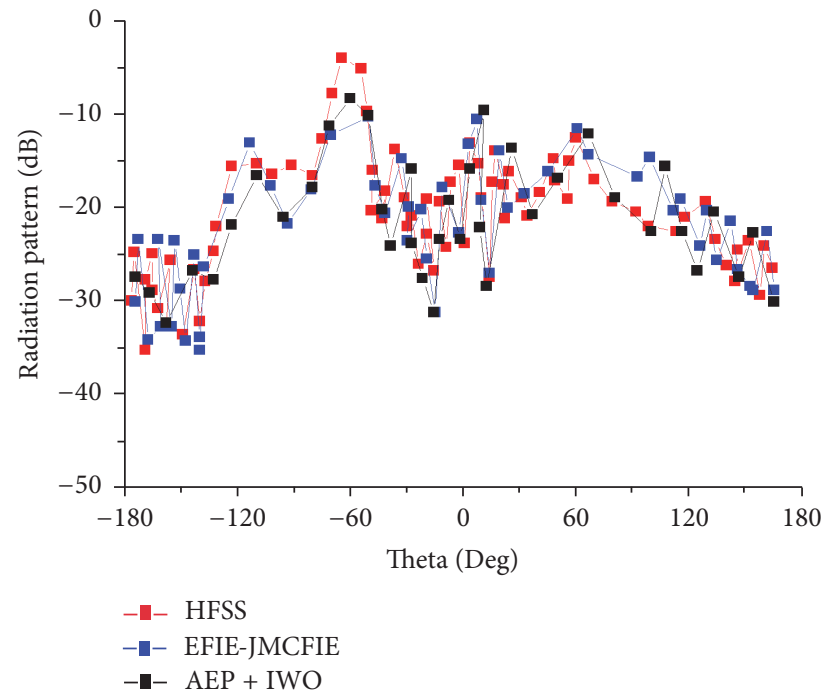

(c) Scanning angle is $60^{\circ}$

FIGURE 21: Radiation pattern of the optimized array antenna in K-band.

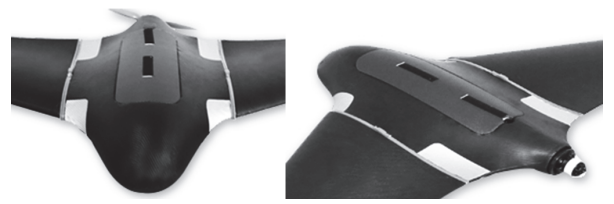

Figure 22: Antenna processing diagram.

\section{Conclusion}

In this paper, the design of the common antenna and its array is based on structure of UAV carrier. As for cone or cylinder carrier, it first adopts the AEP and IWO to make optimization design on the traditional square patch antenna array; the related processing and testing on the designed antenna are performed, which illustrates the AEP combined with the comprehensive application of conformal array IWO and their excellent performance. Then based on the antenna 


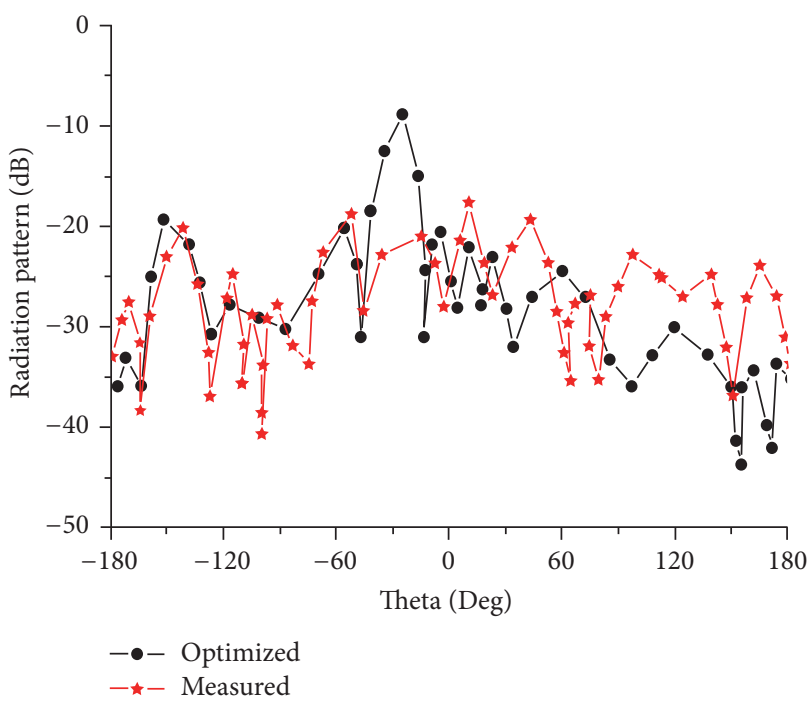

(a) Scanning angle is $-30^{\circ}$

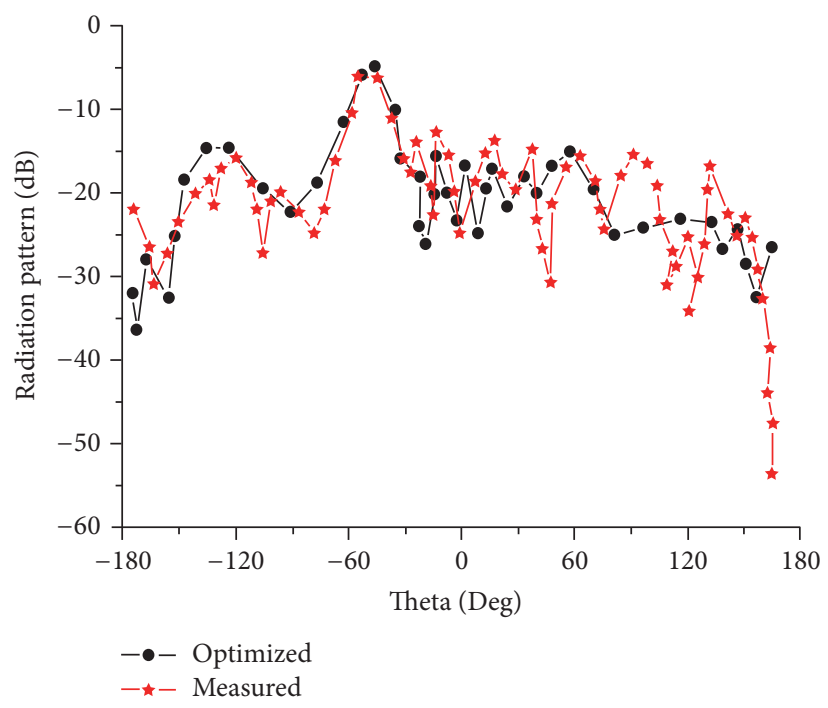

(b) Scanning angle is $30^{\circ}$

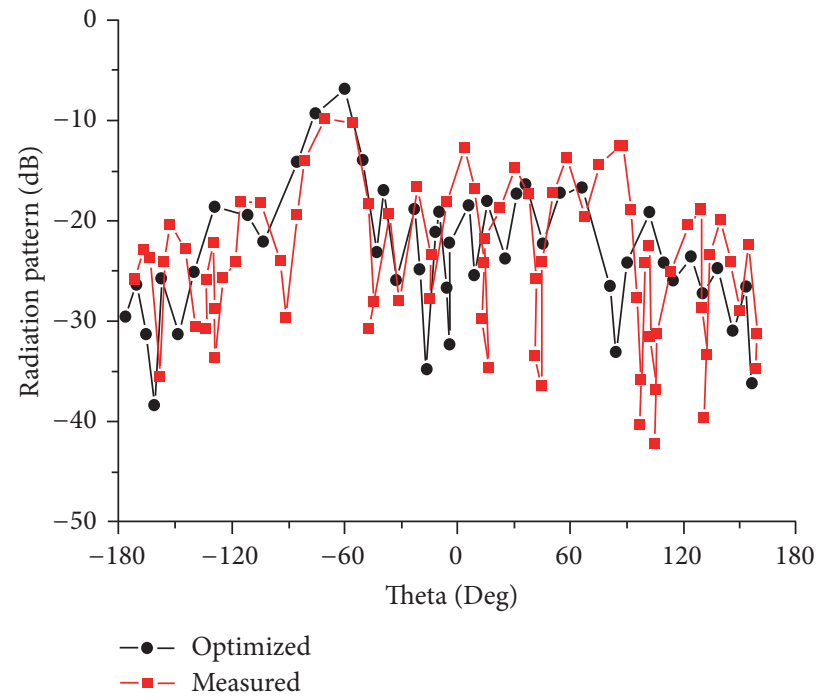

(c) Scanning angle is $60^{\circ}$

FIGURE 23: Radiation pattern of array antenna in the X-band.

TABLE 1: Distribution of antenna array in X-band.

\begin{tabular}{lccc}
\hline Number & \multicolumn{3}{c}{$\begin{array}{c}\text { Scan angle } \\
\text { Phase }\left(30^{\circ}\right)\end{array}$} \\
\hline$(1)$ & Phase $\left(15^{\circ}\right)$ & 35.713 & -123.816 \\
$(2)$ & 30.213 & -72.33 & 52.1334 \\
$(3)$ & -88.123 & 150.78 & -110.23 \\
$(4)$ & 173.453 & 26.098 & 89.44 \\
$(5)$ & 91.09 & -92.96 & -64.5895 \\
$(6)$ & 21.234 & 126.6008 & 135.8254 \\
$(7)$ & -86.076 & -18.3002 & -26.1899 \\
$(8)$ & 180 & 180 & 177.8862 \\
\hline
\end{tabular}

unit miniaturization technology, it designs out a wide angle scanning array; its array beam scanning angle can reach about $75^{\circ}$; the test results show that, in this way, the design of array can realize the wide angle scanning features. In the case of the cylindrical carrier, the method of using the antenna unit miniaturization and the combination of the series and feed is realized, and the water plane of beam is fully polarized.

As for UAV carried antenna, it designs out the conformal vertical polarization antenna unit, and the EFIE-JMCFIE and MLFMA are combined together in the analysis of two different features of UAV carrier, and the test results and analysis of the results are in good agreement.

We also design out a total of $\mathrm{X} / \mathrm{K}$ aperture array, using the AEP-IWO to make optimization, and EFIE-JMCFIE 
TABLE 2: Optimized K-band antenna array feed phase distribution.

\begin{tabular}{lccc}
\hline Number & & Scan angle & \\
& Phase $\left(15^{\circ}\right)$ & Phase $\left(30^{\circ}\right)$ & Phase $\left(60^{\circ}\right)$ \\
\hline$(1)$ & 21.43 & 19.74 & 106.19 \\
$(2)$ & -69.12 & -122.98 & -57.36 \\
$(3)$ & -171.75 & 64.95 & 136.24 \\
$(4)$ & 48.88 & -45.07 & -14.11 \\
$(5)$ & 0.578 & 178 & 160.33 \\
$(6)$ & -81 & 81.08 & 31.45 \\
$(7)$ & 167.22 & -50.151 & -138.513 \\
$(8)$ & 77.618 & 174.798 & 84.23 \\
$(9)$ & -1.0084 & 39.3794 & -63.128 \\
$(10)$ & -109.899 & -91.776 & 156.81 \\
$(11)$ & 163.92 & 141.11 & 3.08 \\
$(12)$ & -46.56 & -89.75 & 7.55 \\
$(13)$ & -56.60 & 140.11 & -141.56 \\
$(14)$ & -131.55 & -25.11 & 30.12 \\
$(15)$ & 141.11 & 140.30 & -141.11 \\
\hline
\end{tabular}

combined with MLFMA are adopted in the radiation characteristics analysis of antenna arrays; the results are in good agreement with those obtained with full-wave simulation software HFSS; the test results also show that the array can scan at a specified beam direction.

\section{Conflicts of Interest}

The authors declare that there are no conflicts of interest.

\section{References}

[1] Z. Lou and J.-M. Jin, "Finite-element analysis of phased-array antennas," Microwave and Optical Technology Letters, vol. 40, no. 6, pp. 490-496, 2004.

[2] Z. Lou and J.-M. Jin, "Higher-order finite element analysis of finite-by-infinite arrays," in Proceedings of the IEEE Antennas and Propagation Society Symposium 2004 Digest held in Conjunction with: USNC/URSI National Radio Science Meeting, pp. 3505-3508, usa, June 2004.

[3] J.-M. Jin and D. J. Riley, "Finite element analysis of antennas and arrays," Finite Element Analysis of Antennas and Arrays, pp. 1435, 2008.

[4] Y.-J. Li and J.-M. Jin, "A new dual-primal domain decomposition approach for finite element simulation of 3-D large-scale electromagnetic problems," Institute of Electrical and Electronics Engineers. Transactions on Antennas and Propagation, vol. 55, no. 10, pp. 2803-2810, 2007.

[5] Y. Li and J.-M. Jin, "A vector dual-primal finite element tearing and interconnecting method for solving 3-D large-scale electromagnetic problems," Institute of Electrical and Electronics Engineers. Transactions on Antennas and Propagation, vol. 54, no. 10, pp. 3000-3009, 2006.

[6] N. M. Mohammad, A. S. Ramezan, G. Mohammad et al., "An elliptical cylindrical FDTD algorithm for modeling conformal patch antenna," IEEE Trans. Antennas Propagation, vol. 58, no. 12, pp. 3990-3996, 2010.

[7] M. V. T. Heckler and A. Dreher, "Analysis of conformal microstrip antennas with the discrete mode matching method," IEEE Transactions on Antennas and Propagation, vol. 59, no. 3, pp. 784-792, 2011.

[8] W.-J. Zhao, L.-W. Li, E.-P. Li, and K. Xiao, "Analysis of radiation characteristics of conformal microstrip arrays using adaptive integral method," Institute of Electrical and Electronics Engineers. Transactions on Antennas and Propagation, vol. 60, no. 2, part 2, pp. 1176-1181, 2012.

[9] M. He and X. Xu, "MoM analysis of conformal antennas based on the volume-surface integral equation," in Proceedings of the 2010 9th International Symposium on Antennas Propagation and EM Theory, ISAPE 2010, pp. 832-835, chn, December 2010.

[10] F. Zhao, K. Xiao, S. L. Chai, Z. B. Liu, and H. Y. Qi, "Analysis of probe-fed microstrip conformal array using VSIE formulation," in Proceedings of the 2012 International Conference on Microwave and Millimeter Wave Technology, ICMMT 2012, pp. 1797-1799, chn, May 2012.

[11] J. Ouyang, Analysis and synthesis of common antenna and array, University of Electronic Science and Technology, Chengdu, cHINA, 2008.

[12] J. Ouyang, X. Luo, and J. Yang, "Analysis and synthesis of conformal conical surface linear phased array with volume surface integration AEP (Active Element Pattern) and INSGAII. IET Micro," Antennas Propagtion, vol. 6, no. 11, pp. 1277-1285, 2012.

[13] P. H. Pathak, P. Janpugdee, and R. J. Burkholder, "A comparison of two different ray based methods for analyzing large convex conformal antenna arrays," in Proceedings of the 4th European Conference on Antennas and Propagation, EuCAP 2010, esp, April 2010.

[14] X.-F. Liu, Y.-C. Jiao, F.-S. Zhang, and Y.-B. Chen, "Design of a conformal microstrip antenna array mounted on an irregular dielectric surface," in Proceedings of the APMC 2005: AsiaPacific Microwave Conference 2005, December 2005.

[15] Houluping., The Analysis And Research of The Microstrip Antenna on The Medium Carrier, University of Electronic Science and Technology, Chengdu, China, 2009. 


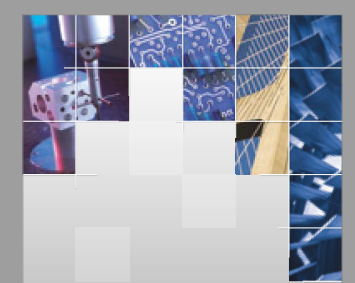

\section{Enfincering}
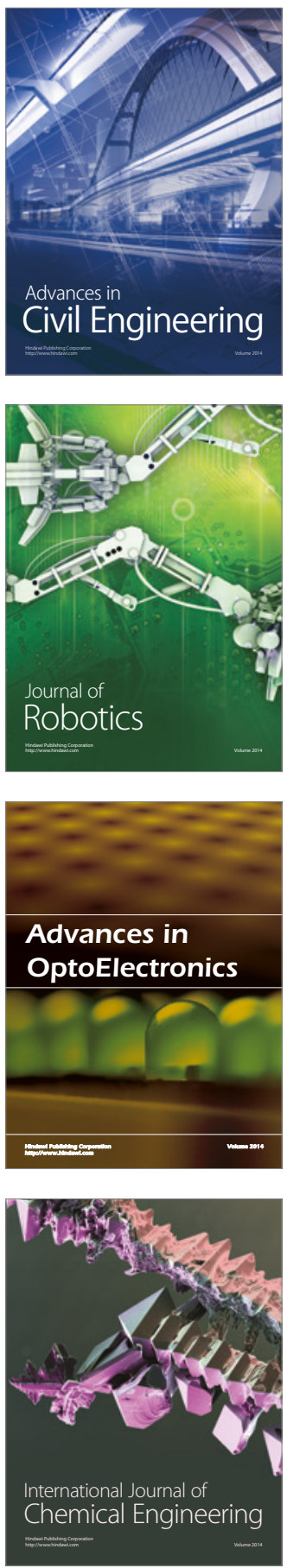

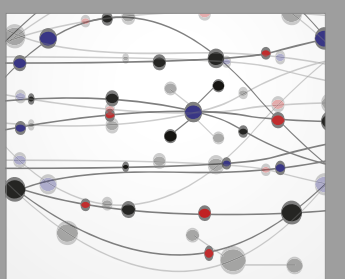

The Scientific World Journal

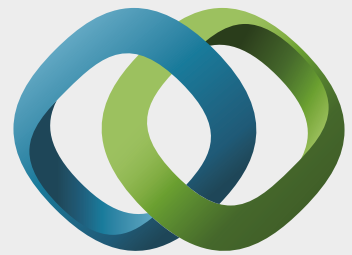

\section{Hindawi}

Submit your manuscripts at

https://www.hindawi.com
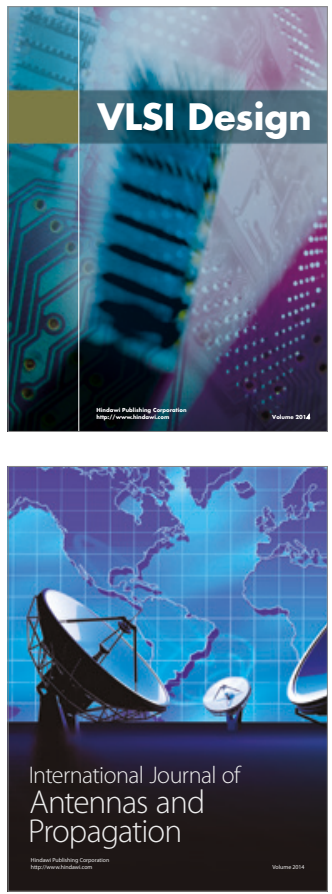

\section{Rotating}

Machinery
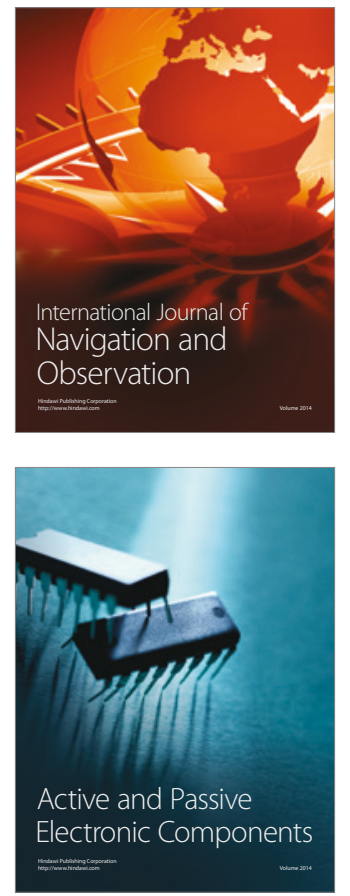
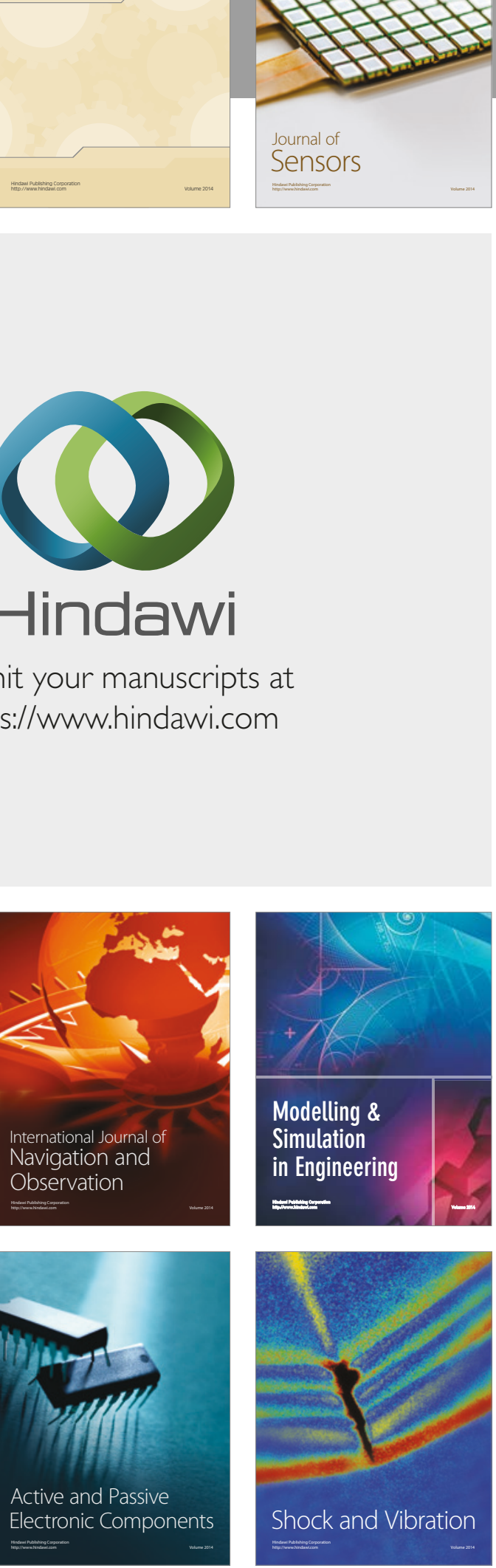
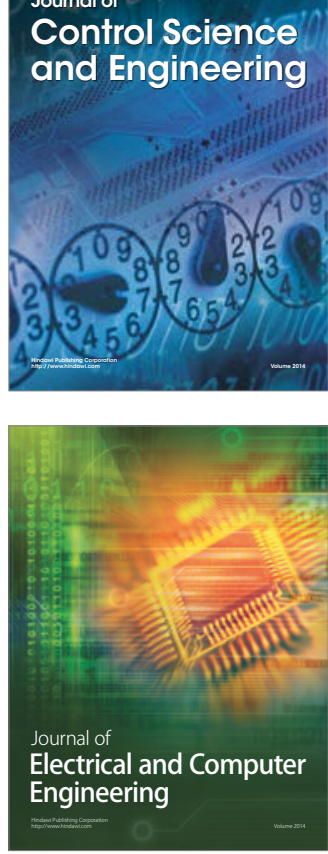

Distributed

Journal of

Control Science

and Engineering
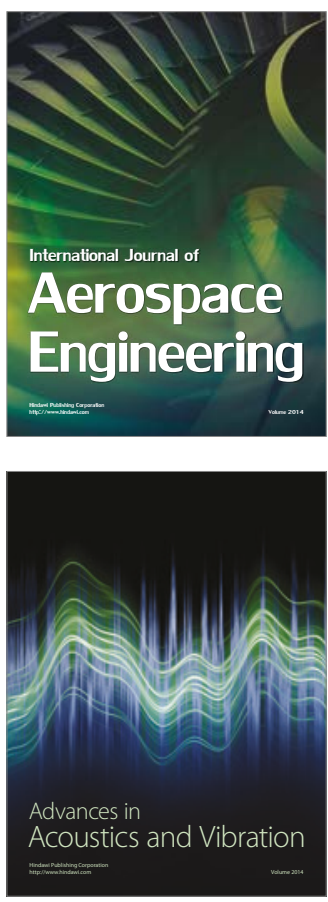

Sensor Networks 